\title{
Uso De Geogebra Y Su Incidencia En El Proceso Enseñanza- Aprendizaje De Grafica De Funciones En El Nivel Superior
}

\section{Coronel Maji Franklin Marcelo}

Magister en Ciencias de la Educación Aprendizaje de la Matemática Docente de la Escuela Superior Politécnica de Chimborazo, Facultad de Informática y Electrónica

\section{Guilcapi Mosquera Jaime Rodrigo}

Magister en Matemática aplicada mención Modelación Matemática y Simulación Numérica, Docente de la Escuela Superior Politécnica de Chimborazo, Facultad de Informática y Electrónica

\section{Vargas Guambo Juan Mario}

Magister en Matemática Básica, Docente de la Escuela Superior Politécnica de Chimborazo, Facultad de Informática y Electrónica

Doi: 10.19044/esj.2018.v14n21p1 URL:http://dx.doi.org/10.19044/esj.2018.v14n21p1

\begin{abstract}
The objective of the research was to determine the incidence of the use of GeoGebra in the learning process of graph of functions of the subject of Mathematics 1 in the students of the first semester of the School of Electronic Engineering in Telecommunications and Networks of the Polytechnic School of Chimborazo. The research is bibliographic, descriptive and quasi-experimental, to which a hypothesis to be demonstrated was proposed. A curricular plan was considered which was developed by a control group (traditional method - 38 students) and an experimental group (use of GeoGebra - 37 students). The groups were evaluated in 2 parts, in the (Pre) and (Post) phases through an evaluation rubric composed of different activities. An intervention plan was applied for the experimental group that allowed to establish an improved scenario to the traditional one. The obtained data from each group represented the academic performance, which was tabulated, analyzed and interpreted. Finally, the results were subjected to a Ztest, which allowed to determine the use of GeoGebra as an academic support tool that has a positive impact on the teaching-learning process of representation of the results of the graphs of functions.
\end{abstract}

Keywords: GeoGebra, Incidence, Teaching-learning, Function Graph 


\section{Resumen}

El objetivo de la investigación fue determinar la incidencia del uso de GeoGebra en el proceso de aprendizaje de gráfica de funciones de la asignatura de Matemáticas 1 en los estudiantes del primer semestre de la Escuela de Ingeniería Electrónica en Telecomunicaciones y Redes de la Escuela Superior Politécnica de Chimborazo. La investigación es bibliográfica, descriptiva y cuasi experimental, a la que se planteó una hipótesis a demostrar. Se consideró un plan curricular que fue desarrollado por un grupo de control (método tradicional - 38 estudiantes) y uno experimental (uso de GeoGebra - 37 estudiantes). Los grupos fueron evaluados en dos momentos un antes (Pre) y un después (Post), a través de una rúbrica de evaluación compuesta de diferentes actividades. Se aplicó un plan de intervención para el grupo experimental que permitió establecer un escenario mejorado al tradicional. Los datos obtenidos de cada grupo, representaron el rendimiento académico alcanzado, mismos que fueron tabulados, analizados e interpretados. Finalmente fueron sometidos a una prueba estadística Z, que permitió determinar que el uso de GeoGebra como herramienta de apoyo académico, incide positivamente en el proceso de enseñanza aprendizaje de representación de resultados de las gráficas de funciones.

Palabras Claves: GeoGebra, Incidencia, Enseñanza-aprendizaje, Grafica de Funciones

\section{Introduction}

Las TICS y su incorporación en las instituciones de educación superior ha dado pasos gigantes, de tal manera que nos permite introducirlas en todo el mundo, proporcionando así la interrelación entre las personas e instituciones a nivel mundial, derrumbando barreras entre el espacio y tiempo.

Sin embargo, tomando en cuenta las nuevas políticas gubernamentales en referencia a la Educación Superior, en diversos países de latino América, son análogas con la finalidad de regular las instituciones educativas, de tal manera de evidenciar posiciones activas frente al reto de estar al día con las tecnologías y establecer así nuevos paradigmas educativos (Gil, Morales \& Basantes, 2014; Tedesco, 2017; Jiménez \& Figueroa, 2017).

En el Ecuador y de manera particular refiriéndonos a las Instituciones de Educación Superior (IES), detectan dificultades para proporcionar un aprendizaje significativo que conduzca al docente desde del lugar que esta al lugar donde el desea estar. (Peñaherrera, 2016).

Si hablamos de tecnología, los docentes expresan que un grupo significativo de docentes -el 30\%-, no hace uso de ella. Este dato concuerda 
con el estudio realizado por Larrea (2013), señalando que existen debilidades en el uso y aplicación de las Tecnologías de la Información y las Comunicaciones (TICS) en los procesos de formación de la educación superior, por lo que, en la mayoría de los casos, el aula es el único ambiente, medio y recurso metodológico para definir las diversas modalidades de aprendizaje.

Dwyer (1974) en su propuesta que es una de las que actualmente tiene mayor difusión en las Instituciones de Educación Superior (IES), ya que se encuentra ligada a los Materiales Educativos Computacionales tales como: algoritmo (el aprendiz es conducido a donde desea llegar de forma secuencial con actividades bien diseñadas) o heurístico (el docente mediante la experiencia debe llegar al conocimiento, creando sus propios modelos de pensamiento) (Panqueva, 1988).

En el caso de las Instituciones de Educación Superior ecuatorianas clasificadas en categoría B, según el Consejo de Evaluación, Acreditación y Aseguramiento de la Calidad de la Educación Superior (CEAACES, 2015a; 2015b), la Escuela Superior Politécnica de Chimborazo (ESPOCH) ha mejorado su oferta académica y rediseños curriculares de las carreras de la Facultad de Electrónica, de manera particular la Escuela de Telecomunicaciones y Redes, pero la metodología de enseñanza con la manifestación del uso de Materiales Educativos Computacionales no se ha aplicado de manera formal ni reglamentada según se propone.

Hohenwarter (2014), eligió GeoGebra con el atenuante que el docente pueda tener una herramienta didáctica que ayude en el proceso de la educación, con las consideraciones que el software a utilizar sea accesible, libre, de fácil manipulación, que cuente con un proceso de instalación automático, sencillo y que sea aceptado en todas las plataformas.

GeoGebra al tener una ventana algebraica, permite ingresar coordenadas y ecuaciones directamente, incluso variables y comandos propios. Es decir, una expresión en la ventana algebraica se corresponde con un objeto de la ventana geométrica, y viceversa; esto evita en cierta medida los problemas que se pueden generar cuando ocurren cambios de representaciones y además permite la traducción de lenguajes: natural y matemático. (Laura \& Gálves, 2009).

GeoGebra es una alternativa utilizada en el ámbito matemático, difundida como herramienta especializada en gráficas de funciones en matemática y se encuentra disponible para el manejo dentro de la Facultad de Electrónica en la Escuela Superior Politécnica del Chimborazo (ESPOCH) y su aplicabilidad para las gráficas de funciones que promueve en los docentes, para la representación de funciones que pueden permitir el incremento de la creatividad y la consolidación de geometría espacial; impulsando, de esta manera, su habilitación como seres autónomos, creativos, críticos y 
emprendedores con capacidades y competencias para actuar y desarrollarse en un contexto cada vez más diverso y desconocido.

García (2014), considera que el GeoGebra es un recurso tecnológico que puede ser utilizado en el saber del aprendizaje y que debe ser incluido en la planificación de una clase como material didáctico para el desarrollo de actividades.

Bonilla (2013), indica que gracias a que GeoGebra permite obtener el resultado del ejercicio de una función de forma rápida y precisa, se lo comienza a emplear después de sustentar la teoría de cada concepto (recta, exponencial) que se detallan en el contenido matemático para verificar los resultados que se obtiene al resolver los ejercicios de forma tradicional.

Márquez (1999), indica que es importante; que un conjunto de técnicas dinámicas sea incluidas como una marca competitiva en la práctica de las matemáticas, pudiendo considerar a GeoGebra ya que es software libre y de fácil manejo que permite trabajar contenidos de geometría, algebra, análisis, calculo, etc.

El objetivo del presente artículo es describir la influencia de la capacitación del uso de GeoGebra como parte del proceso enseñanzaaprendizaje de funciones en el nivel superior de Ingeniería en Telecomunicaciones y Redes. No se consideran estrategias, pues se orientan específicamente en determinar si el uso de GeoGebra incide en el proceso de aprendizaje.

\section{Metodología}

La investigación realizada fue científica, ya que se recogió información relevante del contex to de la investigación. Fue de campo, ya que se interpretó el contexto de la investigación y se determinó una realidad comprobada a través de una prueba estadística hipotética. Se analizó un ambiente representada por una población y muestra que cumplió con el papel de ser la fuente de datos. Es cuantitativa, ya que se evaluó la muestra en dos escenarios a través de un conjunto de evaluaciones contenidas en una rúbrica, que permitió obtener calificaciones para ser comparados mediante una prueba estadística. Fue descriptiva ya que se discuten y se interpretan los datos obtenido. Y finalmente bibliográfica que fundamentó el conocimiento de los contenidos de la guía como técnica a implementarse.

Se utilizó el método factual, puesto que los datos obtenidos son de las pruebas acertadas, que fueron contrastados con hechos reales para poner a prueba una hipótesis. Además, se utilizó el método analítico ya que se estudió por separado cada una de las variables para ver las relaciones existentes entre las mismas.

Los instrumentos de recolección de información fueron definidos bajo una rúbrica revisada por pares académicos para que sean verificados $y$ 
validados. La rúbrica de evaluación fue planificada y programada con un conjunto de actividades de forma sistemática.

Para la demostración de la relación existente entre las dos variables planteadas: el uso manejo de GeoGebra y el aprendizaje de graficas de funciones, fue necesario aplicar una lógica descriptiva y cuasi-experimental en una investigación que reúnen criterios y puntos de vista de los principales entes, mediante los cuales se desarrolla el contexto universitario en estudio.

La población para la presente investigación, fueron los estudiantes de primer semestre la Carrera de Ingeniería en Telecomunicaciones y Redes de la Facultad de Informática y Electrónica en el período académico septiembre 2016 - febrero 2017, que reciben el capítulo de gráficas de funciones, de la asignatura de Matemática 1. De lo cual se tomó una muestra intencionada conformada por dos grupos el de control y el experimental.

$\mathrm{El}$ grupo de control representa el paralelo " $\mathrm{A}$ " conformada por 38 estudiantes a quienes desarrollaron las actividades académicas de forma tradicional; mientras que el grupo experimental representado por el paralelo "B" está conformado por 37 estudiantes quienes desarrollaron el aprendizaje mediante el uso de GeoGebra siendo necesario un laboratorio de cómputo. La asignación del docente de cada grupo se realizó de manera accidental.

Tabla 1.Distribución de los grupos de experimental y de control.

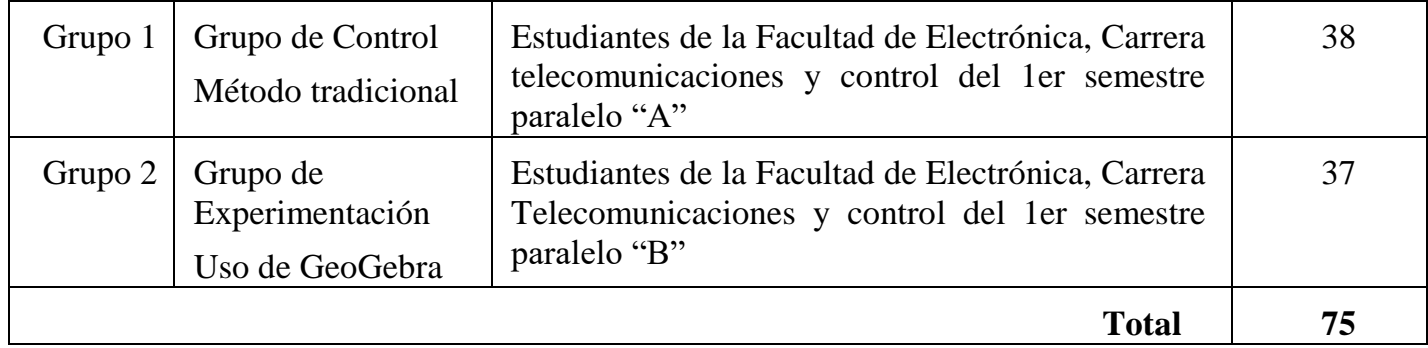

Fuente: Secretaria académica

Elaborado por: Coronel Franklin, 2017

En el grupo experimental la variable disponibilidad hace mención a todos los elementos tecnológicos que el docente debe disponer para que el estudiante tenga acceso al desarrollo de las actividades académicas referentes a los contenidos de la asignatura. La frecuencia de uso de GeoGebra, se registró manera sistemática tanto en el aula, como en el aula virtual y blog. Se consideró una sola asignatura y un mínimo 6 horas semanales (1 encuentro de 3 horas).

Para la investigación se consideraron aspectos importantes tale como:

- GeoGebra como recurso didáctico tecnológico y como herramienta de apoyo pedagógico. 
- Laboratorio de computación adecuado con el software instalado.

- Plan de intervención para el uso adecuado de GeoGebra

- Diagnóstico de facilidad de uso de GeoGebra

- Diagnóstico de GeoGebra como ayuda de entendimiento.

- Motivación en el aprendizaje.

Los parámetros anteriores permitieron que el grupo de experimental, se desenvuelva en un escenario adecuado de aprendizaje, con el fin de que el rendimiento académico sea incidido por la variable independiente.

Se realizó la caracterización de la situación actual de las herramientas tecnológicas con las que dispone el docente para el desempeño académico, de las cuales tenemos las siguientes que se muestran en la Tabla 2.

Tabla 2.Herramientas tecnológicas para uso del docente en las aulas.

\begin{tabular}{|l|l|}
\hline HERRAMIENTA & PROVEEDOR \\
\hline Grupo 1Sistema de Gestión de Aprendizaje (Aulas Virtuales) & MOODLE \\
\hline Blogs(Grupo 1) & Blogger \\
\hline Software matemático (Grupo 1) & GeoGebra \\
\hline Grupo 2 & Tradicional \\
\hline
\end{tabular}

Fuente: Departamento de computo de la Carrera de Telecomunicaciones

Elaborado por: Coronel Franklin, 2017

Las evaluaciones se aplicaron en dos momentos o instantes. La evaluación Pre se aplicó a toda la muestra y representan la evaluación antes de aplicar el método tradicional o el uso de GeoGebra; mientras que el Post, representa la evaluación luego de desarrollar las actividades tradiciones y con el uso de GeoGebra. Lo datos obtenidos fueron procesados a través de una tabulación que permitió realizar un diagnóstico inicial y una evaluación final.

Se desarrolló un plan de capacitación orientado al uso adecuado de GeoGebra, el cual fue aplicado al Grupo Experimental. Una vez aplicado el plan de capacitación se realizó la evaluación post, donde se seleccionaron las mismas preguntas del aspecto uso de GeoGebra realizadas en el momento Pre. Finalmente, se realizó el análisis estadístico de los resultados obtenidos entre el grupo de estudio y el grupo de control.

Para la tabulación de la información, procesamiento de datos y la comprobación de la hipótesis, se utilizó las herramientas informáticas de Microsoft Excel y SPSS, permitiendo visualizar los resultados en tablas y gráficos estadísticos para su análisis e interpretación correspondiente.

Los resultados obtenidos del grupo de control y del grupo experimentan antes y después de aplicar el método tradicional y mediante el uso de GeoGebra para el aprendizaje de las grafica de funciones, fueron sometidos a la prueba estadística $\mathrm{Z}$ a fin de establecer las conclusiones pertinentes. 


\section{Resultados}

Los resultados obtenidos de las evaluaciones aplicadas a los 75 estudiantes que integran el grupo control (38 estudiantes) y el grupo experimental (37 estudiantes) que cursan el primer nivel de la Carrera de Telecomunicaciones.

Las calificaciones obtenidas fueron obtenidas mediante la aplicación de una rúbrica de evaluación sometida a los grupos de control y experimento.

Proponiendo que:

H1; El uso de GeoGebra influencia positivamente en el proceso enseñanzaaprendizaje de grafica de funciones en los estudiantes de primer semestre de la carrera de Telecomunicaciones de la Escuela Superior Politécnica de Chimborazo.

Ho; El uso de GeoGebra no influencia positivamente en el proceso enseñanzaaprendizaje de grafica de funciones en los estudiantes de primer semestre de la carrera de Telecomunicaciones de la Escuela Superior Politécnica de Chimborazo.

Tabla 1. Rendimiento académico del grupo de control

\begin{tabular}{|r|c|c|}
\cline { 2 - 3 } \multicolumn{1}{c|}{} & $\begin{array}{c}\text { MEDIA } \\
\text { ARITMÉTICA }\end{array}$ & $\begin{array}{c}\text { DESVIACIÓN } \\
\text { ESTÁNDAR(S) }\end{array}$ \\
\hline PRE TEST & 4,184 & 1,19 \\
\hline POST TEST & 4,631 & 1,20 \\
\hline PROMEDIO GENERAL & $\mathbf{4 , 4 0 7}$ & $\mathbf{1 , 1 9 5 1}$ \\
\hline
\end{tabular}

Fuente: SPSS

Elaborado por: Franklin Coronel, 2017

Tabla 2. Rendimiento académico del grupo experimental

\begin{tabular}{|r|c|c|}
\cline { 2 - 3 } \multicolumn{1}{c|}{} & $\begin{array}{c}\text { MEDIA } \\
\text { ARITMÉTICA }\end{array}$ & $\begin{array}{c}\text { DESVIACIÓN } \\
\text { ESTÁNDAR(S) }\end{array}$ \\
\hline PRE TEST & 4,35 & 2,17 \\
\hline POST TEST & 8,54 & 1,26 \\
\hline PROMEDIO GENERAL & $\mathbf{6 , 4 4 6}$ & $\mathbf{1 , 7 1 8}$ \\
\hline
\end{tabular}

Fuente: SPSS

Elaborado por: Franklin Coronel, 2017

Tabla 3: Grupo de control vs grupo de experimental

\begin{tabular}{lccc}
\hline GRUPO & N & Promedio & Desviación Estándar \\
\hline Control & 38 & 4,41 & 1,20 \\
Experimental & 37 & 6,45 & 1,72 \\
\hline
\end{tabular}

Fuente: SPSS

Elaborado por: Franklin Coronel, 2017 
Cálculo de la prueba paramétrica $\mathrm{Z}$ se rechaza la hipótesis nula si:

$$
\begin{gathered}
Z_{C}<-Z_{T} \quad Z_{C}<=-1,96 \\
\text { O también } Z_{C}>Z_{T} \quad Z_{C}>=1,96
\end{gathered}
$$

Donde: $Z_{T}$, es el valor teórico de $\mathrm{Z}$

Nivel de significación del $5 \%, \alpha=0,05$;

La investigación tuvo un 95\% de confiabilidad; caso contrario se aceptaba la hipótesis de investigación con una de las dos alternativas.

Los datos fueron:

$$
\begin{aligned}
& \overline{x_{e}}=\mathbf{6 , 4 5} \\
& \overline{x_{c}}=\mathbf{4 , 4 1} \\
& \sigma_{\mathrm{e}}=1,72 \\
& n_{c}=37 \\
& \sigma_{c}=1,20 \\
& \sigma_{\mathrm{c}}=1,20 \quad n_{e}=38 \\
& Z=\frac{\overline{x_{e}}-\overline{x_{c}}}{\sqrt{\frac{\sigma_{e}^{2}}{n_{e}}+\frac{\sigma_{c}^{2}}{n_{c}}}} \\
& Z_{c}=5,94
\end{aligned}
$$

\section{Decisión estadística.}

Al comparar el valor de $\mathrm{Z}$ calculado y el valor de $\mathrm{Z}$ teórico se tuvo que:

$$
\begin{gathered}
Z_{C}>Z_{T} \\
5,94>1,96
\end{gathered}
$$

Se observó que el valor de $Z_{C}=5,95$ se ubica en la zona de rechazo de la hipótesis nula, por lo que se rechazar la hipótesis nula $H_{0}: \bar{x}_{e}=\bar{x}_{C}$ y se aceptó la hipótesis de investigación Hi: $\bar{x}_{e} \neq \bar{x}_{C}$ con la alternativa $\mathrm{A}_{1}: \bar{x}_{e}>$ $\bar{x}_{C}$,

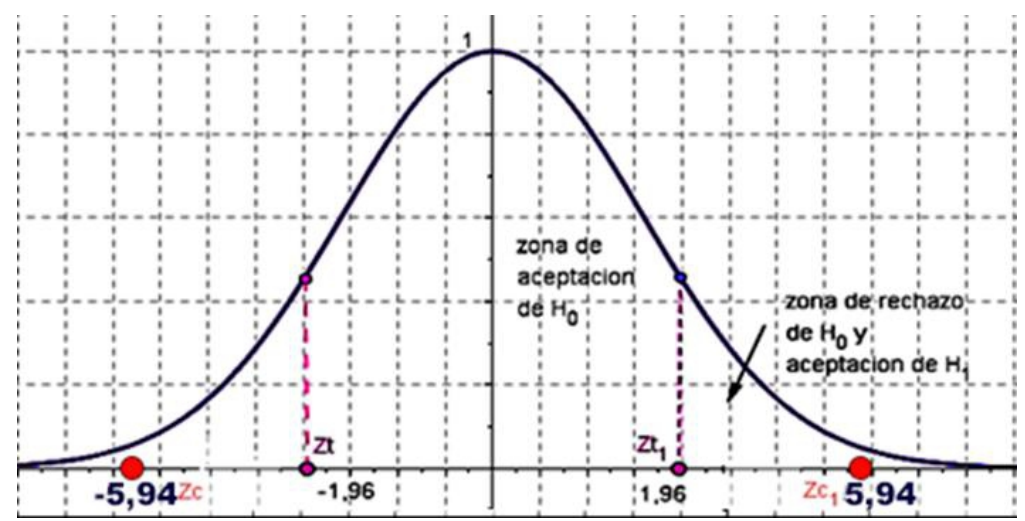

Grafico 1. Representación Gráfica 
Con el nivel de significación de 0,05 equivalente al +/- 1,96, se rechazó la hipótesis nula Ho, pues la solución obtenida de Zc=5,95 se encuentra en el intervalo de la región de rechazo de Ho. Por lo que se acepta la hipótesis alternativa $\mathrm{H} 1$, que indica que el uso de GeoGebra influencia positivamente en el proceso enseñanza- aprendizaje de grafica de funciones en los estudiantes de primer semestre de la carrera de Telecomunicaciones de la Escuela Superior Politécnica de Chimborazo.

El uso de GeoGebra influenció positivamente en el proceso enseñanzaaprendizaje de grafica de funciones en los estudiantes que representan al grupo experimental, como lo demuestra las medias y la comprobación de la hipótesis planteada en la investigación.

\section{Discusión}

GeoGebra es un programa pensado en geometría, álgebra, cálculo y para la ilustración y el saber de las matemáticas. Además de ser intuitivo, fácil de usar, con grandes posibilidades pedagógicas y en continuo desarrollo. (Campo, 2012). Sin duda alguna, se convirtió en una herramienta tecnológica para la enseñanza- aprendizaje de grafica de funciones incide positivamente en el rendimiento académico de los estudiantes, pero se debe considerar que los alumnos en su proceso de aprendizaje deben identificar claramente lo fundamentos conceptuales, algebraicos y de visualización. Así también es importante que se conozca el procedimiento que la herramienta tecnológica automatiza para llegar a la representación gráfica; ya que mucho de ellos son transparente para el usuario.

Se debe indicar también que GeoGebra no tiene una estrategia de resolución clara, pero sin duda el uso de GeoGebra les proporciona un soporte visual con resultado rápido.

Por otro lado, GeoGebra favoreció con sus múltiples representaciones de conceptos geométricos, ayudó a evitar obstáculos algebraicos, permitió centrarse en los conceptos geométricos, así como a resolver los problemas de otra forma.

\section{Conclusiones}

Los contenidos y las actividades que fueron revisadas por pares académicos para el desarrollo de las actividades académicas en la asignatura de matemáticas 1 con respecto a las funciones, establecieron una ruta similar para que los dos grupos, el de control y experimental estudien los mismos contenidos. Es decir que aprendieron los mismos temas, pero con diferentes recursos y materiales didácticos.

El plan de intervención y los recursos que utilizó en grupo experimental denotaron en la incidencia positiva del aprendizaje, reflejados y comprobados 
a través de una prueba estadística, donde se obtuvieron mejores resultados que fueron de la mano con un escenario donde se desenvolvieron.

Como resultado de la investigación realizada se consiguió la caracterización de la situación actual de las herramientas tecnológicas con lenguaje GeoGebra con las que cuenta la institución. El análisis de los criterios y parámetros del uso adecuado del GeoGebra, en la docencia a nivel superior, por parte de los docentes y estudiantes en el proceso de enseñanza de grafica de funciones aplicadas en problemas de ingeniería de Telecomunicaciones, posibilita mejorar las alternativas de enseñanza- aprendizaje.

La consideración de los actores intervinientes, las formas de organización institucional adoptadas y las principales aplicaciones tecnológicas, facilitan el éxito del proceso enseñanza- aprendizaje, es hoy una exigencia consustancial a la educación superior.

Se observó que, en el proceso inicial, los estudiantes tenían problema en el uso de GeoGebra, pero una vez que se ambientaron lograron acelerar el proceso en la resolución e interpretación de los procedimientos, para alcanzar el resultado de las representaciones gráficas de funciones. Por lo que la mayoría de los estudiantes coincidieron que el uso de GeoGebra ayuda a visualizar el problema.

A diferencia de lo estudiantes que siguieron el proceso tradicional de realizar la representación gráfica mediante un proceso tradicional (lápiz y papel), presentaron problemas y dificultades pues los procedimientos para llegar a los resultados requieren de materiales didácticos que no optimizan el tiempo para llegar a la representación gráfica de una función.

\section{References:}

1. Asamblea Nacional de Ecuador. (2008). Constitución de la República. Quito, Ecuador. Recuperado de:

http://www.asambleanacional.gob.ec/es/contenido/constitucion-de-larepublica-del-ecuador

2. Bonilla, G. E. (2013). Influencia de uso del Programa GeoGebra en el Rendimiento Académico en Geometría Analítica Plana (Tercera ed.).

3. CEAACES. (2015a). Resolución No. 130-CEAACES-SE-17-201. Formato autoevaluación IES, Quito, Ecuador.

4. CEAACES. (2015b). Modelo genérico de evaluación del entorno de aprendizaje de carreras presenciales y semipresenciales de las universidades y escuelas politécnicas del Ecuador, Quito, Ecuador.

5. Dwyer, T. A. (1974). Heuristic strategies for using computers to enrich education. International Journal of ManMachine Studies, 6 (2), 137-154. 
6. García, F. A. (2014). Primer Encuentro de Mujeres de Matemáticas. Mexico: Soluciones Empresariales Pantiger y Asociados S.A de C.V Sociendad Matemática Mexicana

7. Gil Álvarez, J. L., Morales Cruz, M. y Basantes Garcés, J. L. (2014). Una aproximación a la calidad universitaria a partir de los procesos de evaluación y acreditación. Universidad y Sociedad, 7 (1), 17-21. Recuperado de: http://rus.ucf.edu.cu/

8. Gómez, D. R. \& Roquet, J. V. (2012). Metodología de la investigación. México, Tlalnepantla: Red Tercer Milenio.

9. Hohenwarter, M. (09 de 12 de 2014). Tesis Escuela de matemática. Documento de ayuda GeoGebra Manual Oficial Version 3,2.

10. Jiménez, A. y Figueroa, H. (2017). Políticas educativas en la educación superior: 1952-1992. Revista Colombiana de Educación, (38-39). http://revistas.pedagogica.edu.co/index.php/RCE/article/view/5439/4 466

11. Laura, C., \& Gálves, C. (2009). Los aprendizajes de los estudiantes de América Latina y el Caribe .

12. Larrea Granados, E. (2013). El currículo de la educación superior desde la complejidad sistémica. Ecuador, Quito: CES.

13. Marqués, P. (1999). TIC aplicadas a la educación. Revista Educar (25), 175-202.

14. Panqueva, A. H. G. (1988). Ambientes de enseñanza-aprendizaje enriquecidos con computador. Boletín de Informática Educativa, 1(2), 117-139.

15. Peñaherrera, M. (2016). Evaluación de un programa de fortalecimiento del aprendizaje basado en el uso de las TIC en el contexto ecuatoriano. Revista Iberoamericana de Evaluación Educativa, 4(2).

16. SENPLADES. (2013). Plan Nacional para el Buen Vivir 2013-2017. Quito, Ecuador.

17. Tedesco, J. C. (2017). Estrategias de desarrollo y educación: el desafío de la gestión pública. Revista colombiana de educación, [S.I], (24). Recuperado http://revistas.pedagogica.edu.co/index.php/RCE/article/view/5203 